\title{
Linear confinement and stress-energy tensor around static quark and anti-quark pair - Lattice simulation with Yang-Mills gradient flow -
}

Ryosuke Yanagihara*, ${ }^{a}$ Takumi Iritani, ${ }^{b}$ Masakiyo Kitazawa,,${ }^{a, c}$ Masayuki Asakawa, ${ }^{a}$ Tetsuo Hatsuda ${ }^{d, b}$ (FlowQCD collaboration)

${ }^{a}$ Department of Physics, Osaka University, Toyonaka, Osaka 560-0043, Japan

${ }^{b}$ RIKEN Nishina Center, RIKEN, Wako, 351-0198, Japan

${ }^{C} J$-PARC Branch, KEK Theory Center, Institute of Particle and Nuclear Studies, KEK, 203-1, Shirakata, Tokai, Ibaraki 319-1106, Japan

${ }^{d}$ RIKEN Interdisciplinary Theoretical and Mathematical Sciences Program (iTHEMS), RIKEN, Wako 351-0198, Japan

E-mail: yanagihara@kern.phys.sci.osaka-u.ac.jp

We study the spatial distribution of the stress tensor around static quark-anti-quark pair in SU(3) lattice gauge theory. In particular, we reveal the transverse structure of the stress tensor distribution in detail by taking the continuum limit. The Yang-Mills gradient flow plays a crucial role to make the stress tensor well-defined and derivable from the numerical simulations on the lattice [1].

The 36th Annual International Symposium on Lattice Field Theory - LATTICE2018

22-28 July, 2018

Michigan State University, East Lansing, Michigan, USA.

\footnotetext{
* Speaker.
} 


\section{Introduction}

The energy-momentum tensor (EMT), $\mathscr{T}_{\mu v}(x)$, is a fundamental observable in physics. Its spatial component is called the stress tensor, $\sigma_{i j}=-\mathscr{T}_{i j}$, which represents the distortion of fields induced by external sources. The Maxwell stress-tensor in electromagnetism is a well-known example: $\sigma_{i j}^{\text {Maxwell }}=-\mathscr{T}_{i j}^{\text {Maxwell }}=-\left(F_{i \mu} F_{j}^{\mu}-\frac{1}{4} \delta_{i j} F_{\mu v}^{2}\right)$ [2]. EMT in Yang-Mills (YM) theory plays a particularly important role because it represents the response of the YM field against external sources in a gauge invariant manner.

In this work [1], we explore the stress distribution around a static quark $(Q)$ and an anti-quark $(\bar{Q})$ separated by length $R$. In YM theory, the field strength is believed to be squeezed into a quasione-dimensional structure called the flux tube and gives rise to the linear confining potential [3]. In previous studies on the structure of the flux tube, the action density and the color electric field have been employed [4-6]. In the present study we perform a first measurement of the stress tensor distribution around static $Q \bar{Q}$ pair. We use the EMT operator on the lattice [7] defined via the YM gradient flow [8], which has been applied extensively to the study on thermodynamics $[9,10]$.

\section{Energy-Momentum Tensor around Static Quark and Anti-Quark}

We first recapitulate the general feature of $\mathscr{T}_{\mu v}(x)$ in the Euclidean spacetime with $\mu, v=$ $1,2,3,4$. The local energy density and the stress tensor read respectively as $\varepsilon(x)=-\mathscr{T}_{44}(x), \sigma_{i j}(x)=$ $-\mathscr{T}_{i j}(x)(i, j=1,2,3)$. The force per unit area $\mathscr{F}_{i}$ is the momentum flow through a given surface element with the normal vector $n_{i}: \mathscr{F}_{i}=\sigma_{i j} n_{j}=-\mathscr{T}_{i j} n_{j}$ [2]. The principal axes of stress tensor is obtained by diagonalized $\mathscr{T}_{i j}$ as $\mathscr{T}_{i j} n_{j}^{(k)}=\lambda_{k} n_{i}^{(k)}(k=1,2,3)$, where $n_{i}^{(k)}$ are the principal axes and the strengths of the force per unit area along $n_{i}^{(k)}$ are given by the absolute values of the eigenvalues, $\lambda_{k}$. The force acting on a test charge is obtained by the surface integral $F_{i}=-\int_{S} \mathscr{T}_{i j} d S_{j}$, where $S$ is a surface surrounding the charge with the surface vector $S_{j}$ oriented outward from $S$.

Next let us review how we obtain $\mathscr{T}_{\mu v}(x)$ non-perturbatively around static $Q \bar{Q}$ on the lattice. First, a static $Q \bar{Q}$ system on the lattice is prepared with the rectangular Wilson loop $W(R, T)$ where $Q \bar{Q}$ locate at $\vec{R}_{ \pm}=(0,0, \pm R / 2)$ and in the temporal interval $[-T / 2, T / 2]$. Second, to define EMT in YM theory we use the YM gradient flow [7,9,10]. The starting point of this method is the YM gradient flow equation $d A_{\mu}(t, x) / d t=-g_{0}^{2} \delta S_{\mathrm{YM}}(t) / \delta A_{\mu}(t, x)$, where $t$ denotes the fictitious 5-th dimensional coordinate [8], and $S_{\mathrm{YM}}(t)$ is composed of $A_{\mu}(t, x)$, whose initial condition at $t=0$ is the ordinary gauge field $A_{\mu}(x)$ in the four dimensional Euclidean space. The gradient flow for positive $t$ leads to cooling of the gauge field within the radius $\sqrt{2 t}$. From the flowed field, the renormalized EMT operator is defined as [7]

$$
\mathscr{T}_{\mu v}^{\mathrm{R}}(x)=\lim _{t \rightarrow 0} \mathscr{T}_{\mu v}(t, x), \quad \mathscr{T}_{\mu v}(t, x)=\frac{U_{\mu v}(t, x)}{\alpha_{U}(t)}+\frac{\delta_{\mu v}}{4 \alpha_{E}(t)}\left[E(t, x)-\langle E(t, x)\rangle_{0}\right] .
$$

Here $E(t, x)=(1 / 4) G_{\mu \nu}^{a}(t, x) G_{\mu \nu}^{a}(t, x)$ and $U_{\mu v}(t, x)=G_{\mu \rho}^{a}(t, x) G_{v \rho}^{a}(t, x)-\delta_{\mu v} E(t, x)$ with the field strength $G_{\mu \nu}^{a}(t, x)$ composed of the flowed gauge field $A_{\mu}(t, x)$. The vacuum expectation value $\left\langle\mathscr{T}_{\mu v}(t, x)\right\rangle_{0}$ is normalized to be zero due to the subtraction of $\langle E(t, x)\rangle_{0}$. We use the next-toleading-order coefficients for $\alpha_{U}(t)$ and $\alpha_{E}(t)$ [7]. The validity and usefulness of this EMT operator have been confirmed via the study on thermodynamic quantities in SU(3) YM theory $[9,10]$. 


\begin{tabular}{|c|c|c|c|c|c|c|c|}
\hline$\beta$ & $a[\mathrm{fm}]$ & $N_{\text {size }}^{4}$ & $N_{\text {conf }}$ & \multicolumn{3}{|c|}{$R / a$} & $T / a$ \\
\hline 6.304 & 0.058 & $48^{4}$ & 140 & 8 & 12 & 16 & 8 \\
\hline 6.465 & 0.046 & $48^{4}$ & 440 & 10 & - & 20 & 10 \\
\hline 6.513 & 0.043 & $48^{4}$ & 600 & - & 16 & - & 10 \\
\hline 6.600 & 0.038 & $48^{4}$ & 1,500 & 12 & 18 & 24 & 12 \\
\hline 6.819 & 0.029 & $64^{4}$ & 1,000 & 16 & 24 & 32 & 16 \\
\hline \multicolumn{4}{|c|}{$R[\mathrm{fm}]$} & 0.46 & 0.69 & 0.92 & \\
\hline
\end{tabular}

Table 1: Simulation parameters on the lattice [1]. Spatial size of the Wilson loop $R$ is shown in the lattice and physical units. Temporal size of the Wilson loop is set to $T$ unless otherwise stated.

The expectation value of $\mathscr{T}_{\mu v}(t, x)$ around the $Q \bar{Q}$ is obtained by [11]

$$
\left\langle\mathscr{T}_{\mu v}(t, x)\right\rangle_{Q \bar{Q}}=\lim _{T \rightarrow \infty} \frac{\left\langle\mathscr{T}_{\mu v}(t, x) W(R, T)\right\rangle_{0}}{\langle W(R, T)\rangle_{0}}
$$

where $T \rightarrow \infty$ is to pick up the ground state of $Q \bar{Q}$. The measurements of $\mathscr{T}_{\mu v}(t, x)$ for different values of $t$ are made at the mid temporal plane $x_{\mu}=\left(\vec{x}, x_{4}=0\right)$, while $W(R, T)$ is defined at $t=0$.

Finally, we obtain the renormalized EMT distribution around $Q \bar{Q}$ from the lattice data by taking the double limit, $\left\langle\mathscr{T}_{\mu v}^{\mathrm{R}}(x)\right\rangle_{Q \bar{Q}}=\lim _{t \rightarrow 0} \lim _{a \rightarrow 0}\left\langle\mathscr{T}_{\mu v}(t, x)\right\rangle_{Q \bar{Q}}^{\text {lat }}[9,10]$. In lattice simulations we measure $\left\langle\mathscr{T}_{\mu v}(t, x)\right\rangle_{Q \bar{Q}}^{\text {lat }}$ at finite $t$ and $a$, and make an extrapolation to $(t, a)=(0,0)$ according to the formula [10], $\left\langle\mathscr{T}_{\mu v}(t, x)\right\rangle_{Q \bar{Q}}^{\text {at }} \simeq\left\langle\mathscr{T}_{\mu v}^{\mathrm{R}}(x)\right\rangle_{Q \bar{Q}}+b_{\mu v}(t) a^{2}+c_{\mu v} t$, where $b_{\mu v}(t)$ and $c_{\mu v}$ are contributions from lattice discretization effects and the dimension six operators, respectively.

\section{Setup}

We have performed the numerical simulations in SU(3) YM theory on the four-dimensional Euclidean lattice with the Wilson gauge action and the periodic boundary condition. The setup for the simulation is summarized in Table 1. In the measurement of the Wilson loop $W(R, T)$, we adopt the standard APE smearing for each spatial link [12] to enhance the overlap between $W(R, T)$ and the $Q \bar{Q}$ ground state. For the noise reduction, we also adopt the standard multi-hit procedure by replacing every temporal links $[4,13]$.

We consider three $Q \bar{Q}$ distances $(R=0.46,0.69,0.92 \mathrm{fm})$ shown in Table 1 , which are comparable to the typical scale of strong interaction. While the largest $R$ is half the spatial lattice extent $a N_{\text {size }}$ for the two finest lattice spacings, the finite size effects are known to be well suppressed even with this setting [4]. A measure of the ground state saturation in the $Q \bar{Q}$ system is characterized by $P(R, T)=C_{0}(R) e^{-V(R) T} /\langle W(R, T)\rangle_{0}$ with the ground-state potential $V(R)$ and the ground-state overlap $C_{0}(R)$ obtained at large $T$ [4]. The data at $a=0.038 \mathrm{fm}$ shows $|1-P(R, T)|<0.5 \%$ as long as $T>0.19 \mathrm{fm}$ for all $R$ in Table 1. Employing $T \simeq 0.46 \mathrm{fm}$ as shown in the last column of Table 1 ensures the ground state saturation of the Wilson loop. In order to avoid the discritization effect and the over-smearing of the gradient flow [10], one has to choose an appropriate window of $t$ satisfying the condition $a / 2 \lesssim \rho \lesssim L$, where $\rho \equiv \sqrt{2 t}$ is the flow radius and $L \equiv \min \left(\left|\vec{x}-\vec{R}_{+}\right|,\left|\vec{x}-\vec{R}_{-}\right|, T / 2\right)$ is the minimal distance between $x_{\mu}=(\vec{x}, 0)$ and the Wilson loop. 

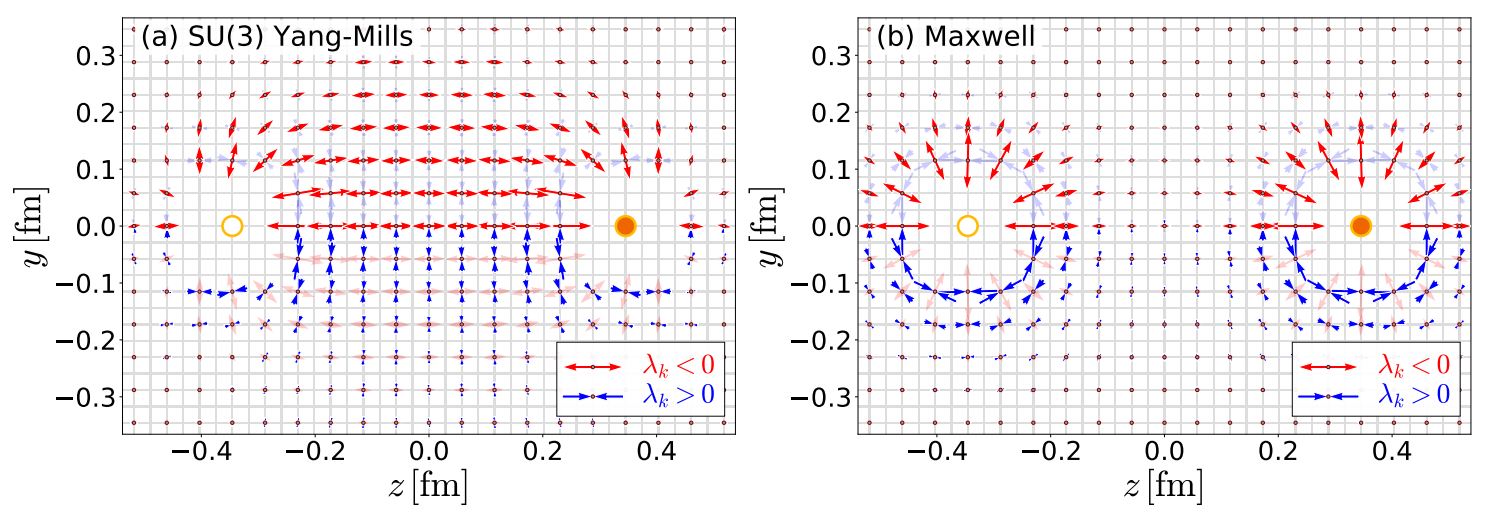

Figure 1: (a) Distribution of the principal axes of $\mathscr{T}_{i j}$ for a $Q \bar{Q}$ system separated by $R=0.69 \mathrm{fm}$ in $\mathrm{SU}(3)$ Yang-Mills theory with $a=0.029 \mathrm{fm}$ and $t / a^{2}=2.0$ [1]. (b) Distribution of the principal axes of the $\mathscr{T}_{i j}$ in classical electrodynamics between opposite charges. In both figures, the red (blue) arrows in the upper (lower) half plane are highlighted.
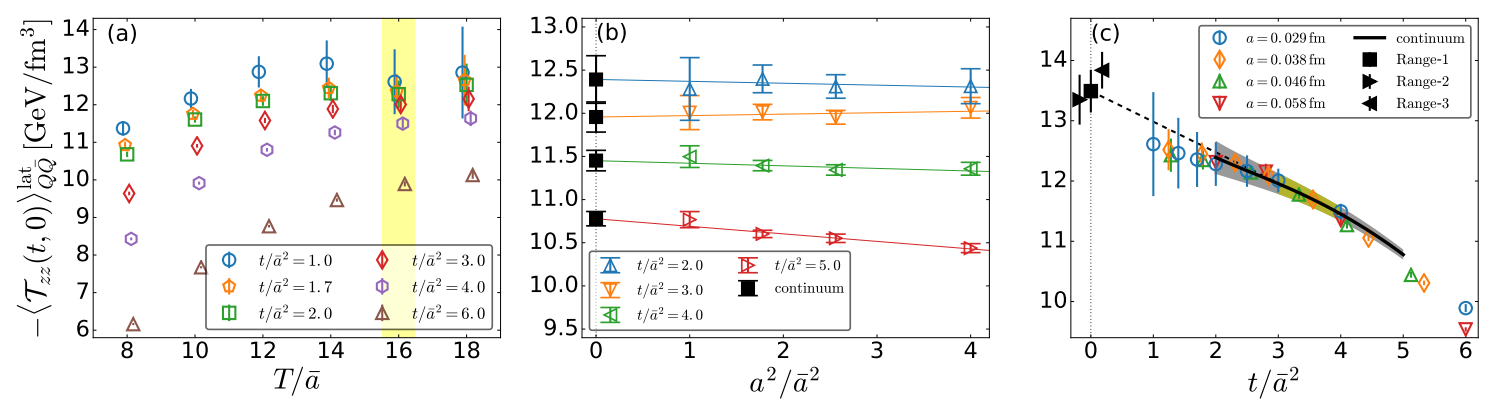

Figure 2: $z z$ component of the stress tensor at $\vec{x}=0$, for various $a$ and $t$ with $R=0.46$ fm [1]. (a) $\left\langle\mathscr{T}_{z z}(t, 0)\right\rangle_{Q \bar{Q}}^{\text {lat }}$ as a function of $T / \bar{a}$ with $a=\bar{a}=0.029 \mathrm{fm}$. To take the double limit, the data at $T / \bar{a}=16$ indicated by the yellow band is used. (b) Open symbols with errors denote $\left\langle\mathscr{T}_{z z}(t, 0)\right\rangle_{Q \bar{Q}}^{\text {lat }}$ as a function of $a^{2} / \bar{a}^{2}$. The filled black symbols are the results of the $a \rightarrow 0$ limit for each $t$. (c) Open symbols with errors are $\left\langle\mathscr{T}_{z z}(t, 0)\right\rangle_{Q \bar{Q}}^{\text {lat }}$ as a function of $t / \bar{a}^{2}$ for different $a$. The solid line corresponds to the result of $a \rightarrow 0$ limit in the interval $2 \leq t / \bar{a}^{2} \leq 5$ with shaded band being statistical error. The filled black symbols are the results of the $t \rightarrow 0$ extrapolation from three different ranges of $t$.

\section{Stress Distribution on the Plane including Two Sources}

Here, we consider the stress distribution on the plane including two charges. Shown in Fig. 1 (a) is the two eigenvectors of the local stress tensor around the two charges separated by $R=0.69 \mathrm{fm}$ obtained on the finest lattice with $a=\bar{a} \equiv 0.029 \mathrm{fm}$ with fixed $t / a^{2}=2.0$. The other eigenvector is perpendicular to the $y-z$ plane. The eigenvector with negative (positive) eigenvalue is denoted by the red outward (blue inward) arrow with its length proportional to $\sqrt{\left|\lambda_{k}\right|}$ :

$$
\hookleftarrow: \lambda_{k}<0, \quad \rightarrow \circ \leftarrow: \lambda_{k}>0
$$

Neighbouring volume elements are pulling (pushing) with each other along the direction of red (blue) arrow. The spatial regions near $Q$ and $\bar{Q}$, which would suffer from over-smearing, are excluded in Fig. 1. The direct analysis of the stress tensor clearly reveals the spatial structure of the 


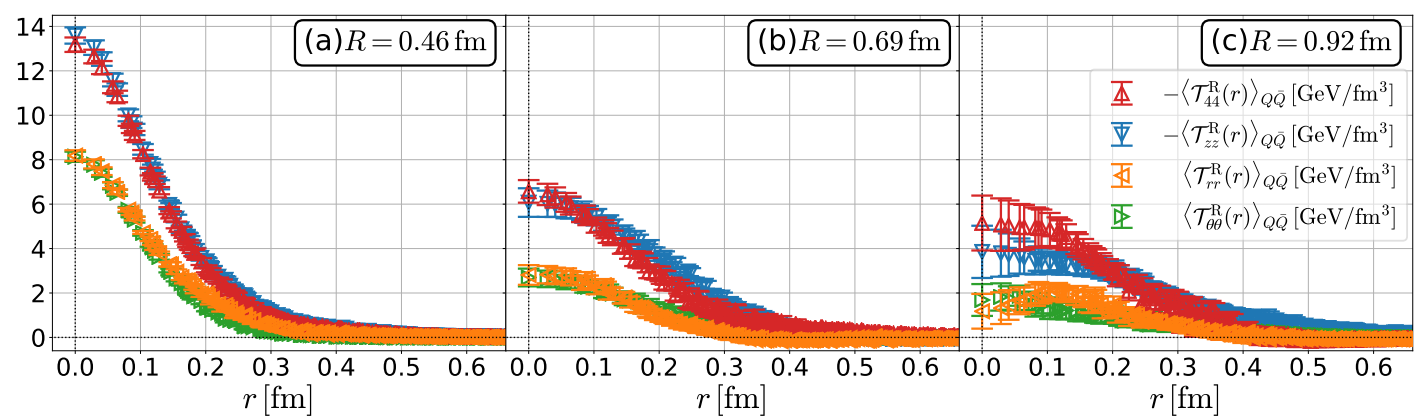

Figure 3: EMT distribution on the mid-plane after the double limit $-\left\langle\mathscr{T}_{c c}^{\mathrm{R}}(r)\right\rangle_{Q \bar{Q}}$ and $-\left\langle\mathscr{T}_{44}^{\mathrm{R}}(r)\right\rangle_{Q \bar{Q}}$ in the cylindrical coordinate system for three different values of the $Q \bar{Q}$ distance $R$ [1].

flux tube in a gauge invariant manner. This is in contrast with the case of the classical electrodynamics with opposite charges shown in Fig. 1 (b).

\section{Stress Distribution on the Mid-Plane between Two Sources}

Let us turn to the mid-plane between $Q$ and $\bar{Q}$. As we take the double extrapolation in the following analysis, we first show this procedure focusing on the mid point $\vec{x}=0$ as an example. Fig. 2 (a) shows the $T$ dependence of $\left\langle\mathscr{T}_{z z}(t, 0)\right\rangle_{Q \bar{Q}}^{\text {lat }}$ for several values of $t$ with $a=0.029 \mathrm{fm}(\equiv \bar{a})$. The figure indicates that there exists a plateau at large $T$, while it becomes narrower as $t$ becomes larger and disappears at $t / \bar{a}^{2} \sim 6$ (i.e., $\rho \sim \sqrt{12} \bar{a}=0.10 \mathrm{fm}$ ). The existence of the plateau means the ground state saturation, while its violation comes from the over-smearing effect. From Fig. 2, we thus conclude that the over-smearing effect is not severe with $T=16 \bar{a}=0.46 \mathrm{fm}$ at $t / \bar{a}^{2} \leq 5$. The same conclusion applies to all the other lattice spacings in Table 1. On the other hand, the lattice discretization effect is non-negligible due to the under smearing at $t / \bar{a}^{2}=2$ ( $\rho=a=0.058$ $\mathrm{fm})$ on the coarsest lattice. Therefore, in the following analysis, we focus on the data in the interval $2 \leq t / \bar{a}^{2} \leq 5(2 \bar{a} \leq \rho \leq \sqrt{10} \bar{a})$, which satisfies $a / 2 \lesssim \rho \lesssim L$ with margin.

In Fig. 2 (b), we show the $a^{2} / \bar{a}^{2}$ dependence of $\left\langle\mathscr{T}_{z z}(t, 0)\right\rangle_{Q \bar{Q}}^{\text {lat }}$ for various $t$ by the open triangles. Here, we have taken the continuum limit with fixed $t$ which is shown by the filled black squares.

In Fig. 2 (c), we show the values of $\left\langle\mathscr{T}_{z z}(t, 0)\right\rangle_{Q \bar{Q}}^{\text {lat }}$ for various values of $a$ and $t / \bar{a}^{2}$ by the open symbols, and the result of the continuum extrapolation in the interval $2 \leq t / \bar{a}^{2} \leq 5$ by the black solid line with the shaded error band. The $t \rightarrow 0$ limit is carried out by using the values in the continuum limit. We have carried out the $t \rightarrow 0$ extrapolation with three fitting windows in order to estimate the systematic errors: $3 \leq t / \bar{a}^{2} \leq 4$ (Range-1), $2 \leq t / \bar{a}^{2} \leq 4$ (Range-2) and $3 \leq t / \bar{a}^{2} \leq 5$ (Range-3). The final results of $\left\langle\mathscr{T}_{z z}^{\mathrm{R}}(0)\right\rangle_{Q \bar{Q}}$ after the double limit are shown by the filled black symbols. The dashed line represents the extrapolation with Range-1.

Let us now turn to the whole mid-plane. We use the cylindrical coordinate system $c=$ $(r, \theta, z)$ with $r=\sqrt{x^{2}+y^{2}}$ and $0 \leq \theta<2 \pi$. On the mid-plane, because of the cylindrical symmetry and the parity symmetry with regard to $z$ axis, EMT is diagonalized as $\left\langle\mathscr{T}_{c c^{\prime}}(t, x)\right\rangle_{Q \bar{Q}}^{\text {lat }}=$ $\operatorname{diag}\left(\left\langle\mathscr{T}_{r r}(t, r)\right\rangle_{Q \bar{Q}}^{\text {lat }},\left\langle\mathscr{T}_{\theta \theta}(t, r)\right\rangle_{Q \bar{Q}}^{\text {lat }},\left\langle\mathscr{T}_{z z}(t, r)\right\rangle_{Q \bar{Q}}^{\text {lat }}\right)$. To take the continuum limit, we need the data at the same $r$ for different $a$. We consider the values of $r$ at which the lattice data are available on the finest lattice. To obtain the data at these $r$ on lattices with other $a$, we interpolate the lattice data 


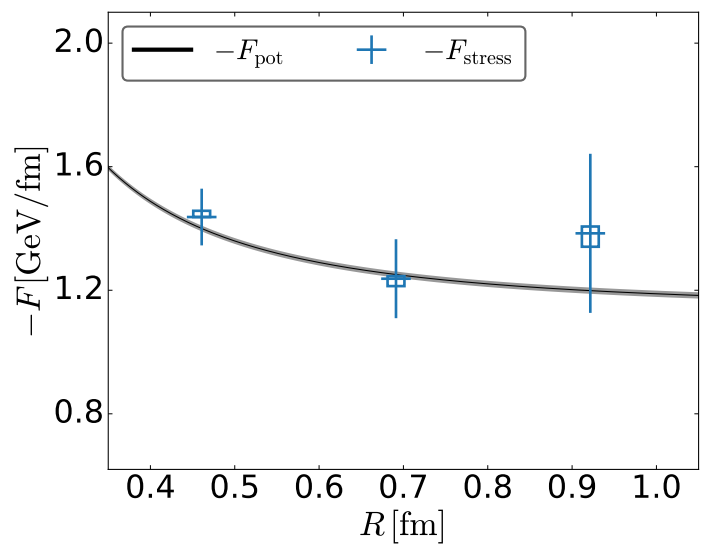

Figure 4: $R$ dependence of the $Q \bar{Q}$ forces, $-F_{\text {stress }}$ and $-F_{\text {pot }}$ [1]. Error bars and rectangular boxes for the latter represent the statistical and systematic errors, respectively.

$\left\langle\mathscr{T}_{c c}(t, r)\right\rangle_{Q \bar{Q}}^{\text {lat }}$ and $\left\langle\mathscr{T}_{44}(t, r)\right\rangle_{Q \bar{Q}}^{\text {lat }}$ with the commonly used functions to parametrize the transverse profile of the flux tube: $f_{\text {Bessel }}(r)=A_{0} K_{0}\left(\sqrt{B r^{2}+C}\right)$ with the 0th-order modified Bessel function $K_{0}(x)$ [14] and $f_{\exp }(r)=\left(A_{0}+A_{1} r^{2}\right) e^{\left(-2 \sqrt{r^{2}+B^{2}}+2 B\right) / C}$ [6]. The $t \rightarrow 0$ limit is taken in the same way as explained above.

In Fig. 3, we show the $r$ dependence of the resulting EMT. From the figure, one finds several noticeable features: (i) Approximate degeneracy between temporal and longitudinal components is found for a wide range of $r:\left\langle\mathscr{T}_{44}^{\mathrm{R}}(r)\right\rangle_{Q \bar{Q}} \simeq\left\langle\mathscr{T}_{Z Z}^{\mathrm{R}}(r)\right\rangle_{Q \bar{Q}}<0$. We also find $\left\langle\mathscr{T}_{r r}^{\mathrm{R}}(r)\right\rangle_{Q \bar{Q}} \simeq$ $\left\langle\mathscr{T}_{\theta \theta}^{\mathrm{R}}(r)\right\rangle_{Q \bar{Q}}>0$, which does not have an obvious reason except at $r=0$. (ii) The scale symmetry broken in the YM vacuum (the trace anomaly) is partially restored inside the flux tube, which is related to the non-zero trace anomaly, $\left\langle\mathscr{T}_{\mu \mu}^{\mathrm{R}}(r)\right\rangle_{Q \bar{Q}}=\left\langle\mathscr{T}_{44}^{\mathrm{R}}(r)+\mathscr{T}_{z z}^{\mathrm{R}}(r)+\mathscr{T}_{r r}^{\mathrm{R}}(r)+\mathscr{T}_{\theta \theta}^{\mathrm{R}}(r)\right\rangle_{Q \bar{Q}}<0$. This is in sharp contrast to the case of classical electrodynamics; $\mathscr{T}_{44}(r)=\mathscr{T}_{z z}(r)=-\mathscr{T}_{r r}(r)=$ $-\mathscr{T}_{\theta \theta}(r)$ and $\mathscr{T}_{\mu \mu}(r)=0$ for all $r$. (iii) Each component of EMT at $r=0$ becomes smaller as $R$ becomes larger, while the transverse radius of the flux tube, typically about $0.2 \mathrm{fm}$, seems to be wide for large $R[6,11]$, although the statistics is not enough to discuss the radius quantitatively.

Finally, we show a non-trivial consistency check of our analysis. We can define the force acting on the charge located at $z>0$ in two different ways, which should give the same result. First, the force is defined from the $Q \bar{Q}$ potential as $F_{\text {pot }}=-d V(R) / d R$. Second, one can also obtain the force by the surface integral of the stress-tensor surrounding the charge, $F_{\text {stress }}=-\int\left\langle\mathscr{T}_{z j}(x)\right\rangle_{Q \bar{Q}} d S_{j}$. For $F_{\text {pot }}$, we fit the numerical data of $V(R)$ calculated from the Wilson loop at $a=0.038 \mathrm{fm}$ with the Cornell parametrization. Note that this lattice spacing is already close to the continuum limit [3]. For $F_{\text {stress }}$, the surface integral is performed on the mid-plane: $F_{\text {stress }}=2 \pi \int_{0}^{\infty}\left\langle\mathscr{T}_{z z}(r)\right\rangle_{Q \bar{Q}} r d r$. Here $\left\langle\mathscr{T}_{z z}(r)\right\rangle_{Q \bar{Q}}$ is obtained by fitting the data in Fig. 3 with either $f_{\text {Bessel }}(r)$ or $f_{\exp }(r)$. In Fig. 4, $-F_{\text {pot }}$ and $-F_{\text {stress }}$ thus obtained are shown by the solid line and the horizontal bars, respectively. For $-F_{\text {stress }}$, we take into account not only the statistical error but also the systematic errors from the double limit and the choice of fitting function, $f_{\text {Bessel,exp }}(r)$. The agreement between the two quantities within the errors is a first numerical evidence that the "action-at-a-distance" $Q \bar{Q}$ force can be described by the local properties of the stress tensor in YM theory. 


\section{Summary and Outlook}

We have explored the spatial distribution of EMT around the $Q \bar{Q}$ system in SU(3) lattice gauge theory. The YM gradient flow plays a crucial role to define EMT on the lattice. We have investigated the stress-tensor distribution on the mid plane by taking the double limit. The linear confining behavior of the $Q \bar{Q}$ potential at long distances is obtained by the surface integral of the stress tensor.

There are interesting applications of this study, such as the generalization to full QCD with the QCD flow equation [16] and the analyses of the $Q Q Q$ system and the $Q \bar{Q}$ system at finite temperature.

\section{Acknowledgement}

The numerical simulation was carried out on IBM System Blue Gene Solution at KEK under its Large-Scale Simulation Program (No. 16/17-07). This work was supported by JSPS Grant-inAid for Scientific Researches, 17K05442, 25287066 and 18H05236.

\section{References}

[1] R. Yanagihara, T. Iritani, M. Kitazawa, M. Asakawa and T. Hatsuda, Phys. Lett. B 789, 210 (2019) doi:10.1016/j.physletb.2018.09.067 [arXiv:1803.05656 [hep-lat]].

[2] L. D. Landau and E. M. Lifshitz, "The Classical Theory of Fields” (fourth Edition) §32, §33, §35 (Butterworth-Heinemann, 1980).

[3] G. S. Bali, Phys. Rept. 343, 1 (2001). J. Greensite, Lect. Notes. Phys. 821, 1 (2011); K. I. Kondo, S. Kato, A. Shibata, and T. Shinohara, Phys. Rept. 579, 1 (2015).

[4] G. S. Bali, K. Schilling, and C. Schlichter, Phys. Rev. D 51, 5165 (1995).

[5] P. Cea, L. Cosmai, and A. Papa, Phys. Rev. D 86, 054501 (2012).

[6] N. Cardoso, M. Cardoso, and P. Bicudo, Phys. Rev. D 88, 054504 (2013).

[7] H. Suzuki, PTEP 2013, no. 8, 083 B03 (2013) [Erratum: PTEP 2015, no. 7, 079201 (2015)].

[8] M. Lüscher, JHEP 1008, 071 (2010); M. Lüscher and P. Weisz, JHEP 1102, 051 (2011).

[9] M. Asakawa et al. [FlowQCD Collaboration], Phys. Rev. D 90, 011501 (2014) [Erratum: Phys. Rev. D 92, no. 5, 059902 (2015)].

[10] M. Kitazawa, T. Iritani, M. Asakawa, T. Hatsuda, and H. Suzuki, Phys. Rev. D 94, 114512 (2016); M. Kitazawa, T. Iritani, M. Asakawa, and T. Hatsuda, Phys. Rev. D 96, 111502 (2017).

[11] M. Lüscher, G. Münster, P. Weisz, Nucl. Phys. B180, 1 (1981).

[12] M. Albanese et al. [APE Collaboration], Phys. Lett. B 192, 163 (1987).

[13] G. Parisi, R. Petronzio, and F. Rapuano, Phys. Lett. 128B, 418 (1983).

[14] J. R. Clem, J. Low Temp. Phys. 18, 427 (1975).

[15] P. Cea, L. Cosmai, F. Cuteri, and A. Papa, JHEP 1606, 033 (2016).

[16] H. Makino and H. Suzuki, PTEP 2014, no. 6, 063B02 (2014) [Erratum: PTEP 2015, no. 7, 079202 (2015)]; Y. Taniguchi, S. Ejiri, R. Iwami, K. Kanaya, M. Kitazawa, H. Suzuki, T. Umeda, and N. Wakabayashi, Phys. Rev. D 96, 014509 (2017). 